\title{
Epithelioid schwannoma or ossifying fibromyxoid tumour?
}

\author{
Ömer Alıcı ${ }^{\text {**, Tuğrul Kesicioğlub }}$ \\ ${ }^{a}$ Department of Pathology, Samsun Training and Research Hospital, Samsun, Turkey \\ ${ }^{b}$ Department of General Surgery, Faculty of Medicine, Ondokuz Mayis Univesity, Samsun, Turkey
}

ARTICLE INFO ABSTRACT

\section{Article History}

Received $\quad 13 / 02 / 2013$

Accepted $\quad 31 / 03 / 2013$

\section{* Correspondence to:}

Ömer Alıc1

Department of Pathology,

Samsun Training and Research Hospital,

Samsun, Turkey,

e-mail: omralc@gmail.com

\section{Keywords:}

Epithelioid

Fibromyxoid

Ossifying

Schwannoma

Tumour

\begin{abstract}
There is a risk of confusing epithelioid schwannoma and ossifying fibromyxoid tumours due to their similar morphological and immunohistochemical features. Epithelioid schwannoma can be described as a benign nerve sheath tumour of peripheral nerves. Ossifying fibromyxoid tumour is considered to be a intermediate malignant soft tissue tumour with the potential for recurrence and metastasis. Because the prognosis of these two tumoral entities are different from each other differential diagnosis is important In this article, our purpose is to present a case, on what we initially considered to be epithelioid schwannoma. However, we later observed, based on the data in the literature, that the tumour appeared to be an ossifying fibromyxoid tumour.
\end{abstract}

J. Exp. Clin. Med., 2013; 30:193-195

\section{Introduction}

Both epithelioid schwannoma and ossifying fibromyxoid tumour (OFMT) have recently been defined as two different entities, often confused with each other due to their similar morphological and immunohistochemical features (Kindblom et al., 1998). While epithelioid schwannoma can be described as a benign nerve sheath tumour of peripheral nerves, OFMT is considered to be a intermediate (borderline) malignant soft tissue tumour. There are differing opinions regarding the origin of the tumour (Kindblom et al., 1998; Miettinen et al., 2008).

The purpose of this article is to present a case, on who we initially considered to be epithelioid schwannoma. However, we later observed, based on the data in the literature, that the tumour appeared to be an OFMT, so we had to change the diagnosis.

\section{Case}

A 47-year-old female patient had an operation at another centre to treat a painless mass on her shoulder of $3 \mathrm{~cm}$ in diameter, which she had for 10 years. It was reported in the pathological evaluation made at the centre that the mass was considered to be a mesenchymal tumour, and the patient was referred to our hospital for immunohistochemical analysis in order to get a differential diagnosis. On examination of H\&E sections made up of paraffin-embedded blocks of the mass, we found a neoplastic structure with high cellularity, comprising morphological cells (mainly epithelioid with some spindles) forming solid, trabecular areas, cord-like structures and accumulations in a collagenase, fibromyxoid stroma. The uniform appearance of the tumour cells was a significant finding. There were cell-rich cellular areas in the tumour, as well as hypocellular, hyalinised fibrotic areas. The walls of some parts of the vascular structures had a hyalinised appearance. In some sections of the tumour periphery, small lamellar bone spicules appeared to surround the mass in a fibrotic, capsule-like structure (Fig. 1A, D). There was no conspicuously significant pleomorphism or atypia in the tumour cells. Mitotic count was $0-1$ in 10 BBA, and no necrosis was observed. There was no lymphovascular invasion. Tumour cells were diffusely and strongly stained positive by S100 and vimentin (Fig 2A, B). Negative staining occurred in the case of smooth muscle actin (SMA), desmine, pancytokeratin, CD34, HMB45 and CD68. The Ki-67 prolifera- 

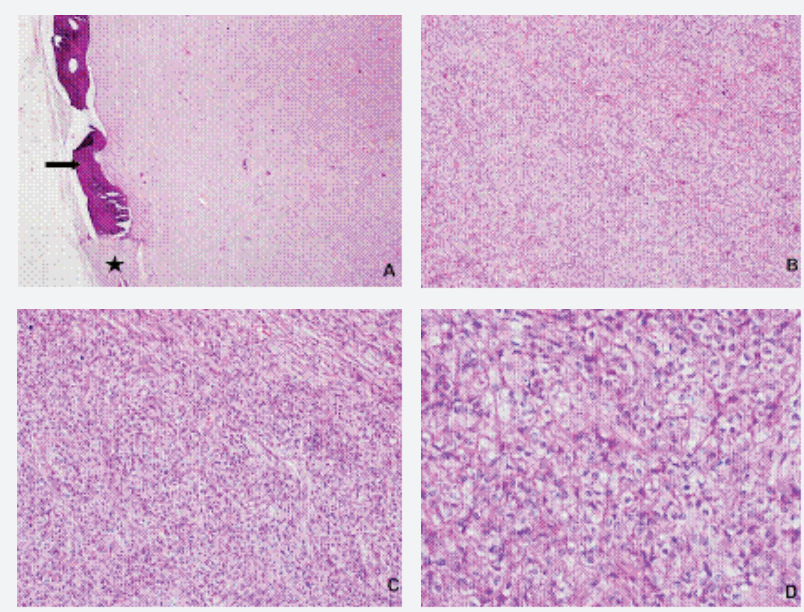

Fig. 1. Ossifying fibromyxoid tumour. A, The fibrous pseudocapsule structure (star) and partially ossification rim (arrow) can be seen at the periphery of the mass (H\&E, $x$ 40). B, C, D, Note the epithelioid morphology and uniform appearance of the tumour cells (H\&E, x 100; H\&E, x 200; H\&E, x 400).


Fig. 2. Immunohistochemical features of OFT. A, Diffuse nuclear and/or cytoplasmic staining with S-100 protein (DAB, x 40). B, Tumor cells are highlighting positive for vimentin (DAB, $x 40)$. C, The Ki-67 proliferation index is lower than $5 \%(C$; DAB, x 200). tion index were lower than 5\% (Fig. 2C). No peripheral nerve section was found in the vicinity of the tumour. The tumour was located at a distance of $1 \mathrm{~mm}$ from the point closest to the surgical margin. The patient underwent a surgical margin reexcision, but no tumour was found during an examination of the re-excision material. Neither local relapse nor metastasis was apparent during the 12-month follow-up period, and no additional treatment was given.

\section{Discussion}

OFMT is a rare soft tissue tumour that was first defined by Enzinger et al. (1989). Although it was initially considered to be benign, in later years it was reported to have rare metastatic potential, and was classified among intermediate malignant soft tissue tumours (Weis and Goldblum, 2008). Epithelioid schwannoma was defined by Orosz et al. (1993) However, they considered that entity to be a non-ossifying form of fibromyxoid tumour. Epithelioid schwannoma was documented and defined comprehensively by Kindblom et al. for the first time in 1998.

OFMT usually occurs in middle-aged individuals, in the upper extremities of the body in particular. It is a large, solid and well-defined lesion, usually painless, and is located in subcutaneous nodules. Although rare, OFMT has been reported in children also. It is more prevalent in men than in women. The tumour is typically $3-5 \mathrm{~cm}$ in diameter, but tumours of larger dimensions have been reported. It has a long pre-operative history in patients, which may last decades (Miettinen et al., 2008; Weis and Goldblum, 2008). In $80 \%$ of the lesions, there is radiological evidence of an incomplete ring of calcification in the peripheral area. The tumour is morphologically characterised by the formation of solid trabecular areas, cord-like structures and accumulations of oval or round uniform cells (epithelioid and spindle in character) in a collagenase and myxoid stroma. There is a thick, fibrous pseudocapsule around the tumour. Most cases show an incomplete lamellar bone ring in the peripheral area. Most commonly, the tumour cells are strongly stained positive by S100 protein and vimentin. Positive staining by desmin, cytokeratin, epithelial membrane antigen (EMA), SMA, glial fibrillary acidic protein (GFAP) or CD10 is rarely reported (Miettinen et al., 2008; Weis and Goldblum, 2008, Graham et al., 2011).

Unlike classic schwannoma, the diagnosis of epithelioid schwannoma may be problematic due to its increased cellularity and distinct epithelioid morphology. Classic schwannoma symptoms such as Verocay bodies, Antoni A/B areas and hyalinisation on the walls of vascular structures are either not present in the case of epithelioid schwannoma, or can only be found in limited areas (Rezanko et al., 2012). Immunohistochemically, the cells are positive for vimentin and S-100 protein (Laskin et al., 2005; Rezanko et al., 2012).

Our patient had neoplastic structures of encapsulated character composed of epithelioid morphological cells forming solid trabecular areas, cord-like structures and accumulations. The cells were stained positive by S100 protein and vimentin. We initially considered our case to be epithelioid schwannoma due to its similarities to OFMT in terms of morphological and immunohistochemical findings; however, we performed another analysis for incomplete peripheral ossification and found that the tumour cells appeared more uniform than in schwannoma, and that there was no peripheral nerve 
section around the tumour. In addition, instead of the intense nuclear staining by S100 that we saw on the peripheral nerve sheath tumours, our attention was drawn by the presence of cytoplasmic staining, which is more significant. Because these characteristics had already been defined, we reported the case as a typical OFMT.

The similarities in morphological and immunohistochemical features may result in confusion of the two tumours (Kindblom et al., 1998; Miettinen et al., 2008; Laskin et al., 2005). In OFMTs, the presence of a peripheral ossification rim, the uniform appearance of the cells and the absence of a peripheral nerve section are indicative findings in differential diagnosis (Kindblom et al., 1998). In addition to epithelioid schwannoma, other tumour types should be considered in differential diagnosis, including malignant peripheral nerve sheath tumours, osteosarcoma, myoepithelioma and extraskeletal myxoid chondrosarcoma (Miettinen et al., 2008).

Typical, atypical and malignant forms of OFMT have been defined (Folpe and Weiss, 2003). In histological terms, the criteria for malignant tumours are defined as high nuclear grade or high cellularity, and the existence of mitosis greater than 2 in 50 BBA. Folpe and Weiss, (2003) reported that the rate of recurrence was $12 \%$ and $13 \%$ in typical and atypical forms, respectively, and that the rate of metastasis was $4 \%$ and $6 \%$, respectively. They reported the rate of recurrence and metastasis in malignant tumours to be $60 \%$ (Folpe and Weiss, 2003). In their study of 46 cases, Graham et al., (2011) found no local recurrence or metastasis in either typical or atypical cases. They reported that the rate of metastasis in malignant cases was 33\%. In contrast, Miettinen et al. (2008) found no metastasis in 104 cases compared according to histological characteristics, and reported the rate of recurrence as $22 \%$. They found that the recurrence rate was higher in cases where the mitotic count was greater than 2 in 50 BBA, compared to cases with a mitotic count of less than 2 . In addition, they found that no significant relationship existed between other histological findings such as tumour diameter, positive surgical margin, the presence of satellite nodules and the risk of recurrence. Apart from postoperative surgical margin re-excision, we administered no additional treatment to our patient, and found no local relapses or metastasis during the 12-month follow-up period.

Although there is no clear information on the origin of OFMT, some researchers suggest that these tumours are neural in origin on the basis of having similar morphological and immunohistochemical characteristics to neural tumours (Kindblom et al., 1998; Saad et al., 2005). In this sense, the cases defined as epithelioid schwannoma can be seen as having an intermediate morphology that falls between classic schwannoma and OFMT (Kindblom et al., 1998).

Consequently, OFMT can be described as rare, slowly evolving, mesenchymal neoplasia with a risk of recurrence and metastasis. They may be confused in particular with epithelioid schwannoma tumours and various neural, myxoid or chondroid tumours due to their unusual morphology and immunohistochemical features. For this reason, it is important in all cases to carry out differential diagnosis.

\section{REFERENCES}

Enzinger, F.M., Weiss, S.W., Liang, C.Y., 1989. Ossifying fibromyxoid tumor of soft parts. A clinicopathological analysis of 59 cases. Am. J. Surg. Pathol. 13, 817-827.

Folpe, A.L., Weiss, S.W., 2003. Ossifying fibromyxoid tumor of soft parts. A clinicopathologic study of 70 cases with emphasis on atypical and malignant variants. Am. J. Surg. Pathol. 27, 421-431.

Graham, R.P., Dry, S., Li, X., Binder, S., Bahrami, A., Raimondi, S.C., Dogan, A., Chakraborty, S., Souchek, J.J., Folpe, A.L., 2011. Ossifying fibromyxoid tumor of soft parts: A clinicopathologic, proteomic, and genomic study. Am. J. Surg. Pathol. 35, 1615-1625.

Kindblom, L.G, Meis-Kindblom, J.M., Havel, G., Busch, C., 1998. Benign epithelioid schwannoma. Am J Surg Pathol. 22, $762-70$.

Laskin, W.B., Fetsch, J.F., Lasota, J., Miettinen, M., 2005. Benign epithelioid peripheral nerve sheath tumors of the soft tissues: Clinicopathologic spectrum of 33 cases. Am. J. Surg. Pathol. 29, 39-51.

Miettinen, M., Finnell, V., Fetsch, J.F.., 2008. Ossifying fibromyxoid tumor of soft parts-a clinicopathologic and immunohistochemical study of 104 cases with long-term follow-up and a critical review of the literature. Am. J. Surg. Pathol. 32, 996-1005.

Orosz, Z., Sapi, Z., Szentirmay, Z., 1993. Unusual benign neurogenic soft tissue tumour. Epithelioid schwannoma or an ossifying fibromyxoid tumour? Pathol. Res. Pract. 189, 601-605.

Rezanko, T., Sari, A.A., Tunakan, M., Calli, A.O., Altinboga, A.A., 2012. Epithelioid schwannoma of soft tissue: Unusual morphological variant causing a diagnostic dilemma. Ann. Diagn. Pathol. 16, 521-526.

Saad, A.G., Mutema, G.K., Mutasim, D.F., 2005. Benign cutaneous epithelioid Schwannoma: Case report and review of the literature. Am. J. Dermatopathol. 27, 45-47.

Weiss, S.W., Goldblum, J.R., 2008. Soft tissue tumors of intermediate malignancy of uncertain type. Weiss \& Goldblum: Enzinger and Weiss's Soft Tissue Tumors. $5^{\text {th }}$ ed. Mosby Elsevier, Philadelphia, pp. 1093-1101. 